\title{
Systemic lupus erythematosus
}

\author{
Authors: Maliha F Shaikh, ${ }^{A}$ Natasha Jordan ${ }^{B}$ and David P D'Cruz ${ }^{C}$
}

Systemic lupus erythematosus (SLE) is a chronic multisystem autoimmune disease that is highly heterogeneous in its presentation. This can pose significant challenges for physicians responsible for the diagnosis and treatment of such patients. SLE arises from a combination of genetic, epigenetic and environmental factors. Pathologically, the disease is primarily driven by loss of immune tolerance and abnormal B- and T-cell function. Major organ involvement may lead to significant morbidity and mortality. Classification criteria for SLE have been developed largely for research purposes; however, these are also widely used in clinical practice. Antinuclear antibodies are the hallmark serological feature, occurring in over $95 \%$ of patients with SLE at some point during their disease. The mainstay of treatment is antimalarial drugs such as hydroxychloroquine, combined with corticosteroids and conventional immunosuppressive drugs. An increasing understanding of pathogenesis has facilitated a move towards the development of targeted biologic therapies, with the introduction of rituximab and belimumab into clinical practice.

\section{Introduction}

Systemic lupus erythematosus (SLE) is a chronic multisystem autoimmune rheumatic disease in which disease flares are interspersed with episodes of remission. In contrast to organ specific autoimmune diseases, SLE comprises a constellation of signs and symptoms that can affect multiple organ systems. This diversity of presentation, changing clinical features and fluctuating disease course often presents challenges in diagnosis and management.

The prevalence of SLE is variable, dependent on ethnic origin and ranges from $40-200$ per 100,000 of population. SLE is more common in those of African and Asian ancestry than in Europeans. It is predominantly a disease of females, with a female to male ratio of 9:1. ${ }^{1-3}$ Non-European patients with SLE tend to have a younger onset of disease and greater incidence of serious organ involvement, reflective of a more severe clinical phenotype. The broad spectrum of clinical presentations

Authors: ${ }^{\text {A }}$ linical research fellow, Department of Rheumatology, Addenbrooke's Hospital, Cambridge, UK; ${ }^{B}$ consultant rheumatologist, Department of Rheumatology, Addenbrooke's Hospital, Cambridge, UK; C professor and consultant rheumatologist, Louis Coote Lupus Unit, Guy's and St Thomas' Hospital NHS Foundation Trust, London, UK includes mucocutaneous, musculoskeletal, haematological, cardiopulmonary, renal and central nervous system manifestations. Lupus nephritis and neuropsychiatric lupus are considered the most severe forms of organ involvement and can result in significantly reduced life expectancy. This has led to recognition of the need for early intensive immunosuppression. Current treatment regimens consist of antimalarial drugs, corticosteroids, conventional disease-modifying anti-rheumatic drugs, cyclophosphamide and biologics. However, conventional therapies fail to adequately suppress disease activity in a significant proportion of patients and newer targeted therapies are being developed to address this unmet need.

\section{Aetiology}

While there is no single causative agent, the disease arises from a complex interplay of genetic, epigenetic and environmental factors. Genome wide association studies have identified over 60 risk loci involved in the susceptibility of SLE. ${ }^{4}$ Monozygotic twin studies show incomplete concordance in the development of SLE, indicating that there are other influences on susceptibility. ${ }^{5}$ Epigenetics, inherited changes in gene expression apart from alterations in the sequence of DNA bases that affect the phenotype, also influence the risk of SLE development. This includes DNA methylation, microRNA regulation and post-translational modification of histones. ${ }^{6}$

\section{Key points}

SLE is highly heterogeneous and may mimic many other diseases

Most patients with SLE will demonstrate ANA positivity at some point during their disease

All patients with SLE should be treated with antimalarials such as hydroxychloroquine, unless contraindicated

Corticosteroids should be used at the lowest effective dose for the shortest duration of time to avoid long-term complications

Comorbidities such as cardiovascular risk, infections and osteoporosis must be addressed in all patients

KEYWORDS: belimumab, hydroxychloroquine, rituximab, systemic lupus erythematosus 
Genetic factors alone are insufficient to explain the onset of SLE and there is likely to be an interaction with environmental factors for the disease to develop in a genetically susceptible individual. Environmental triggers include ultraviolet light, demethylating drugs and viruses. ${ }^{7}$ Sunlight is the most common trigger for a flare of disease, especially cutaneous manifestations. Epstein-Barr virus infection may be an environmental risk factor for the development of SLE, especially in juveniles. Autoantibodies in the sera can precede the development of clinical features of lupus by many years. Chronic Epstein-Barr virus infection may cause interferon alpha production, which is a feature of SLE. ${ }^{8}$ Many drugs, especially those that undergo acetylation such as hydralazine and procainamide, can cause drug-induced lupus, which is usually self-limiting and regresses on withdrawal of the drug. The majority of these patients do not develop SLE. ${ }^{9}$

\section{Immunology}

SLE is the result of multiple defects in both the innate and adaptive immune system with altered immune tolerance, hyperactivation of T- and B-cells, reduced ability to clear immune complexes and apoptotic cells and the failure of multiple regulatory mechanisms within the immune network..$^{10}$ There is abnormal release of intracellular antigens, for example because of dysregulated apoptosis. A loss of B-cell self-tolerance results in excessive autoantibody production with immune complex mediated type III hypersensitivity reactions. Immune complex deposition within tissues leads to complement activation, inflammatory cell recruitment and tissue damage. Innate immune cells are recruited and produce pathogenic cytokines, such as interferon alpha, tumour necrosis factor and interleukin-1 with abnormal type-1 interferon regulation. ${ }^{11}$ B-cell involvement, independent of antibody production, may also occur through antigen presentation, T-cell activation and dendritic cell modulation. ${ }^{11} \mathrm{~T}$-cells also contribute to autoimmunity in SLE with defects associated with CD8+ and T-regulatory cell function occurring along with an expanded $\mathrm{CD} 3^{+} \mathrm{CD} 4^{-} \mathrm{CD} 8^{-} \mathrm{T}$-cell lineage.

\section{Serology}

Antinuclear antibodies (ANA) are the hallmark serological feature of SLE, with the majority (>95\%) of patients having a positive ANA at some point during their disease course. Therefore, ANA is a useful screening test for SLE. There is a strong consensus that the presence of autoantibodies is essential to make a diagnosis of SLE. Enzyme-linked immunosorbent assay (ELISA) tests are increasingly used to test for ANAs but this has a higher risk of a false negative result. A negative ANA on ELISA in a patient with a strong clinical suspicion of SLE should be followed by repeat testing with indirect immunofluorescence to Hep2 cells. ANA negative patients are unlikely to demonstrate positive extractable nuclear antigen (ENA) tests. ${ }^{12}$

False positive ANA results are common secondary to infection or other autoimmune disease. A modestly raised ANA titre may also be seen in individuals with no discernible disease. ${ }^{13}$ Therefore, a positive ANA result should precipitate testing for anti-double stranded DNA (anti-dsDNA) antibodies and antibodies to specific ENAs such as anti-Ro, anti-La, anti-Sm,
anti-RNP. Anti-Ro and anti-La antibodies occur in around $30 \%$ and $20 \%$ of patients with SLE, respectively, although they are more common in Sjögren's syndrome. Flares of disease are often associated with increasing anti-dsDNA antibody titres and decreases in complement levels. Testing for a combination of ANA with anti-dsDNA, ENA (Ro/La/Sm/RNP) and hypocomplementaemia will detect most patients with SLE. ${ }^{14}$ Anti-phospholipid antibodies may occur in conjunction with SLE, or independently, and form part of the classification criteria for SLE. ${ }^{15}$

Complement is commonly used as a marker of disease activity in SLE. In active disease, serum complement levels are usually reduced. A reduction in the levels of components $(\mathrm{Clq}, \mathrm{C} 2$, C4) of the classical pathway is usually seen and there is often a corresponding decrease in C3 levels, especially in severe disease. ${ }^{16}$ In some patients, however, individual complement components may only fluctuate slightly with disease activity and C4 may also remain low during remission. This may be due to the relative rates of complement synthesis and catabolism: hypercatabolism of C3 may be associated with increased or decreased synthetic rates. ${ }^{16}$ Patients with persistent hypocomplementaemia may have a complement deficiency and they are at greater risk of developing serious infection with encapsulated organisms, such as Streptococcus pneumonia and Neisseria meningitidis. The hypocomplementaemia causes defective opsonisation and local phagocytosis but also reduces splenic clearance of these organisms, rendering patients 'functionally asplenic'. Prophylactic antibiotics may be considered in such patients.

\section{Clinical features}

SLE is notorious for its heterogeneity in presentation and its ability to mimic other diseases. A variety of organ systems can be involved (Table 1). Common findings are skin lesions, such as malar rash or discoid lesions, photosensitivity, scarring or non-scarring patchy alopecia, mucocutaneous ulcers, polyarticular arthritis, nephritis with secondary hypertension and pedal oedema, and serositis manifesting as pleuropericarditis with pleural and pericardial effusions.

Although the disease predominantly affects young to middle-aged females, SLE can also present in older age groups, when it often follows a more indolent course. Neuropsychiatric lupus usually occurs in the first 10 years following diagnosis of SLE.

Although the disease is far more common in women than men, the disease can follow a more aggressive course in males, with men demonstrating higher mortality rates than women. ${ }^{17}$ Anti-phospholipid syndrome can occur in association with SLE and is characterised by arterial and venous thromboses and recurrent pregnancy morbidity.

In the development of SLE there may be a pre-clinical phase in which autoantibodies occur in the serum in the absence of any clinical symptoms. Such patients do not require any specific treatment until they develop clinical features of disease. Development of multiple autoantibodies is often followed by inflammation in one or more organs. Recurrent flares of inflammatory disease activity lead to accumulation of damage, which ultimately results in increased morbidity and mortality. In the longer term, comorbidities develop - in particular, accelerated atherosclerosis due to traditional and non-traditional 


\begin{tabular}{|c|c|}
\hline System & Clinical manifestations \\
\hline Constitutional & $\begin{array}{l}\text { Fever, raised inflammatory markers, } \\
\text { lymphadenopathy, fatigue, weight loss }\end{array}$ \\
\hline Dermatological & $\begin{array}{l}\text { Mouth ulcers, malar rash, photosensitivity, } \\
\text { discoid rash, subacute cutaneous lupus, } \\
\text { alopecia, livedo reticularis, purpura, vasculitis, } \\
\text { urticaria }\end{array}$ \\
\hline Musculoskeletal & Arthritis, myositis, arthralgia, myalgia \\
\hline Renal & $\begin{array}{l}\text { Glomerulonephritis, proteinuria, } \\
\text { haematuria, uraemia }\end{array}$ \\
\hline Cardiac & $\begin{array}{l}\text { Libman-Sacks endocarditis, pericarditis, } \\
\text { myocarditis, accelerated atherosclerosis, } \\
\text { hypertension, dyslipidaemia }\end{array}$ \\
\hline Respiratory & $\begin{array}{l}\text { Pleurisy, pleural effusions, interstitial lung } \\
\text { disease, pulmonary hypertension, pulmonary } \\
\text { haemorrhage, pulmonary fibrosis }\end{array}$ \\
\hline Gastroenterological & $\begin{array}{l}\text { Abdominal pain, ascites, hepatitis, } \\
\text { hepatosplenomegaly, peritonitis, clitis }\end{array}$ \\
\hline Neurological & $\begin{array}{l}\text { Seizures, psychosis, headaches, mononeuritis } \\
\text { multiplex, peripheral and cranial neuropathy, } \\
\text { cerebrovascular accident, chorea }\end{array}$ \\
\hline Haematological & Anaemia, thrombocytopenia, leucopenia \\
\hline Vascular & $\begin{array}{l}\text { Raynaud's phenomenon, vasculitis, } \\
\text { thrombosis }\end{array}$ \\
\hline
\end{tabular}

risk factors, osteoporosis, infections and malignancies - which are additional challenges to management. ${ }^{18}$

\section{Diagnosis}

The 1997 American College of Rheumatology (ACR) criteria specify 11 criteria that occur in SLE. In order to be classified with SLE, at least four must be present either serially or simultaneously. In 2012, the revised Systemic Lupus International Collaborating Clinics (SLICC) criteria were proposed (Table 2); this requires the patient to fulfil at least four out of 17 criteria, including at least one of the 11 clinical criteria and one of the six immunological criteria, or have biopsy-proven lupus nephritis in the presence of ANAs or antidsDNA antibodies. The SLICC criteria demonstrate greater sensitivity but lower specificity than the 1997 ACR criteria but may still fail to classify some patients with disease. Both of these classification criteria were designed to be highly specific for research purposes, and should not be used as diagnostic criteria although they are widely used in clinical practice. ${ }^{15}$ Ultimately, a diagnosis of SLE is based on clinical judgement and recognition of patterns of signs and symptoms supported by serological tests and following exclusion of other diagnoses.

\section{Investigations}

As SLE is such a heterogeneous disease, investigations depend on the particular presentation. However, all patients will need blood tests for ANA, ENA, anti-dsDNA and complement as well as
Table 2. Systemic Lupus International Collaborating Clinics criteria (2012)

\section{Clinical criteria}

\section{Immunologic} criteria

Acute cutaneous lupus (malar rash, bullous lupus, toxic epidermal necrolysis Antinuclear antibodies variant of SLE, maculopapular lupus rash, photosensitive lupus rash) or subacute cutaneous lupus (nonindurate psoriaform or annular lesions that

Chronic cutaneous lupus (classic discoid rash, hypertrophic lupus, lupus panniculitis, mucosal lupus, chilblain lupus, discoid lupus/ lichen planus overlap

Oral or nasal ulcers

Non-scarring alopecia

Arthritis

Serositis

Neurologic

Haemolytic anaemia

Leucopenia $\left(<4000 / \mathrm{mm}^{3}\right)$ or lymphopenia $\left(<1000 / \mathrm{mm}^{3}\right)$

Thrombocytopenia $\left(<100,000 / \mathrm{mm}^{3}\right)$

\section{Anti-double} stranded DNA

Anti-Sm

Anti-phospholipid antibodies

Low complement (C3, $\mathrm{C} 4, \mathrm{CH} 50)$

Direct Coombe's test full blood count, which may demonstrate cytopenias, renal and liver function tests and vitamin D levels. Urinalysis should also be performed as a screen for renal involvement and proteinuria quantified, if present. Depending on the exact constellation of symptoms, further investigations - such as radiographs of the hands and feet, musculoskeletal ultrasound, pulmonary function studies, electrocardiograms and echocardiography, chest X-ray and high resolution computerised tomography scan, renal ultrasound and biopsy, magnetic resonance imaging brain and lumbar puncture - may be indicated.

\section{Monitoring}

European League Against Rheumatism recommendations for the management of SLE stipulate that regular monitoring of disease activity is an integral part of treatment. A number of validated disease activity indices, constructed from cohort and cross-sectional studies, are widely used in both clinical practice and clinical trials - such as the British Isles Lupus Assessment Group (BILAG) and the Systemic Lupus Erythematosus Disease Activity Index 2000 (SLEDAI-2K). ${ }^{19}$

Most patients with SLE should be followed up by a physician with expertise in monitoring disease activity and treatment response. Routine clinical monitoring allows earlier detection of active disease. This should include regular checks of blood pressure in order to monitor cardiovascular disease risk and screen for renal disease. Urinalysis for red cells, white cells, proteinuria and cellular casts is useful for detecting clinically unapparent renal disease. If this is abnormal, it should be 
followed with quantification of proteinuria using either a 24-hour urinary protein collection or a protein:creatinine ratio and calculation of glomerular filtration rate. ${ }^{20}$

Full blood count, liver function tests and urea and electrolytes should be routinely monitored in all patients with SLE and especially those on immunosuppressive drugs. Concurrent measures of erythrocyte sedimentation rate and C-reactive protein (CRP) can help to distinguish between a flare of SLE and infection. In disease flares, erythrocyte sedimentation rate is raised with a normal CRP. A raised CRP in SLE is indicative of infection. However, there can be a modest rise in CRP with active serositis or arthritis in the absence of infection. ${ }^{20}$

\section{Treatment}

Treatment of SLE includes the use of antimalarial drugs, corticosteroids, conventional immunosuppression with synthetic immunosuppressive medications, as well as biologic therapies and the detection and management of comorbidities. The aims of treatment are to induce remission of disease flares, and then maintain such remission. The precise treatment administered is dependent on the severity of disease and the organ system involved. Mild SLE can be treated with antimalarials (such as hydroxychloroquine) alone, but often stronger immunosuppression is also required. Non-steroidal antiinflammatory drugs are often considered to be contraindicated because of the increased cardiovascular risks, but very shortterm use for a defined period of time may be acceptable. Management of lupus nephritis is based on early detection and diagnosis followed by treatment usually with mycophenolate mofetil or cyclophosphamide. An increasing understanding of the pathogenesis of SLE and the involvement of B-cells, through both antibody dependent and independent mechanisms, has highlighted the importance of the loss of B-cell self-tolerance in driving autoimmunity in SLE. This has shifted the focus of treatment towards novel, targeted therapies.

\section{Corticosteroids}

Corticosteroids are used as the mainstay of treatment in acute flares of disease and are useful in this context. Often on presentation to acute physicians, especially in the acute setting, patients are started on high-dose steroid therapy that is continued for protracted periods of time. However, their use is associated with significant side effects and in order to limit these, steroids should be used at the lowest effective dose for the shortest duration of time possible. The often quoted 'recommended dose' of $1 \mathrm{mg} / \mathrm{kg} /$ day is arbitrary, non-evidence based and should be avoided in most circumstances. Reduction of steroids is a major goal in the treatment of chronic SLE as evidence is emerging that major damage accumulation results more from steroid therapy than lupus disease activity. ${ }^{21}$ The initial dose of prednisolone in the first month of therapy after diagnosis of SLE is predictive of prednisolone doses over the following 11 months. ${ }^{22}$ Organ complications secondary to chronic corticosteroid use occur in a dose-dependent manner. The most common complications are musculoskeletal damage, such as osteoporotic fracture, and ocular damage, such as cataracts, and these are also among the first complications to occur. An increase in the average prednisolone dose by $1 \mathrm{mg}$ /day is estimated to increase the risk of cataracts by $3.8 \%$ and osteoporotic fractures by $4.2 \% .^{21}$

\section{Conventional immunosuppression}

Conventional immunosuppressive therapies are based around combinations of corticosteroids with azathioprine, mycophenolate mofetil or cyclophosphamide. Although there is a substantial evidence base for their use from multiple clinical trials, especially in lupus nephritis, their use may be limited by adverse effects, bone marrow, gastrointestinal and hepatotoxicity and their failure to sufficiently suppress active disease in certain patients. ${ }^{23,24}$ First-line therapy for all patients and the mainstay of treatment for many patients with SLE are antimalarials such as hydroxychloroquine. These agents have beneficial effects in reducing the frequency and severity of disease flares and thrombotic events, and also influence cardiovascular disease risk. ${ }^{25}$ Methotrexate is often used to treat skin and joint disease. Skin disease may also benefit from topical treatments, such as topical steroids, and topical immunosuppressive agents, such as tacrolimus or pimecrolimus. Patients with SLE should also be encouraged to avoid sun exposure and wear high factor sun block (SPF 50+) as this helps to reduce photosensitivity and cutaneous flares.

\section{Biologic therapies}

The development of biologic therapies in SLE has lagged behind other rheumatological conditions, such as rheumatoid arthritis. However, an increased understanding of the molecular and cellular biology of SLE has led to targeted therapies now becoming available for use. Belimumab is the first drug to be licensed by the US Food and Drug Administration for SLE in 60 years. Belimumab is a humanised monoclonal antibody that inhibits the cytokines B-lymphocyte stimulator or B-cell activating factor (BLyS/BAFF) and a proliferation-inducing ligand (APRIL) thus, targeting B-cells. In two large phase III clinical trials, BLISS 52 and BLISS 72, belimumab demonstrated efficacy in patients with mild to moderate manifestations of SLE, namely musculoskeletal and mucocutaneous disease. ${ }^{26,27}$ Despite a number of negative clinical trials of novel biologic therapies in SLE, a number of ongoing studies are showing promising results, including monoclonal antibodies against type- 1 interferons such as sifalimumab. ${ }^{28}$ In the UK, initial rejection of belimumab by the National Institute for Health and Care Excellence was appealed and it is now approved as an additional therapy in patients with active autoantibody-positive SLE. Patients must demonstrate ongoing serological evidence of active disease with a positive dsDNA antibody level, low complement and a high disease activity score on the Safety of Estrogen in Lupus National Assessment-SLE Disease Activity Index (SELENA-SLEDAI) despite treatment. Treatment with belimumab can only be continued beyond 24 weeks if the SELENA-SLEDAI score improves by four or more points. ${ }^{29}$

Rituximab is a chimeric mouse/human monoclonal antibody to CD20, a B-cell surface antigen. CD20 is a transemembrane cellular phosphoprotein that is expressed by around $95 \%$ of the B-cell population, but not by plasma cells. Rituximab was developed for use in B-cell malignancies and causes selective B-cell depletion. While a large evidence base exists supporting the use of rituximab in SLE, disappointingly, two large clinical trials in lupus nephritis (LUNAR) and non-renal SLE (EXPLORER) failed to meet their primary endpoints. ${ }^{30,31}$ Despite this, there is widespread use of rituximab - particularly 
in those with resistant disease. There are several case series of good response that support the use of rituximab. ${ }^{32-34}$ Further randomised controlled trials of rituximab in SLE are currently underway. In the UK, the use of rituximab in patients with SLE is permitted by NHS England provided data on patient outcomes are captured in the BILAG biologics registry.

\section{Comorbidities}

Coronary artery disease is more common in patients with SLE and aggressive management of conventional risk factors should be routine practice. Hypertension, hyperlipidaemia and diabetes mellitus should all be treated aggressively. Modifiable risk factors, such as smoking, exercise and obesity, should also be addressed. Patients with SLE often have low bone mineral density compared with age-matched healthy controls. Risk factors for osteoporosis should be assessed, including age, menopausal status, history of low trauma fracture, duration and current dose of corticosteroid treatment, family history, diet, smoking, alcohol, weight bearing exercise, malabsorption syndromes and lack of sun exposure. Patients on prolonged courses of corticosteroids should have bone density measured using dual energy X-ray absorptiometry and vitamin D and calcium supplementation is often required.

\section{Conclusions}

Despite numerous failed studies of biologic agents over the past decade, several new therapies are being developed for use in SLE in large scale clinical trials around the world. However, despite recent advances in clinical treatment, SLE remains a huge challenge to clinicians across various disciplines. Mortality in patients with SLE has improved dramatically over the last 50 years but patients still remain at significant risk of premature death due to atherosclerosis and infection. Earlier diagnosis and early aggressive treatment of disease, as well as comorbidities, will help to address this and improve outcomes.

\section{Conflicts of interest}

The authors have no conflicts of interest to declare.

\section{References}

1 Lim SS, Bayakly AR, Helmick CG et al. The incidence and prevalence of systemic lupus erythematosus, 2002-2004: The Georgia Lupus Registry. Arthritis Rheumatol 2014;66:357-68.

2 Feldman CH, Hiraki LT, Liu J et al. Epidemiology and sociodemographics of systemic lupus erythematosus and lupus nephritis among US adults with Medicaid coverage, 2000-2004. Arthritis Rheum 2013;65:753-63.

3 Somers EC, Marder W, Cagnoli P et al. Population-based incidence and prevalence of systemic lupus erythematosus: the Michigan lupus epidemiology and surveillance program: incidence and prevalence of SLE in southeastern Michigan. Arthritis Rheumatol 2014;66:369-78.

4 Teruel M, Alarcón-Riquelme ME. The genetic basis of systemic lupus erythematosus: What are the risk factors and what have we learned. J Autoimmun 2016;74:161-75.

5 Javierre BM, Fernandez AF, Richter J et al. Changes in the pattern of DNA methylation associate with twin discordance in systemic lupus erythematosus. Genome Res 2010;20:170-9.
6 Long H, Yin H, Wang L, Gershwin ME, Lu Q. The critical role of epigenetics in systemic lupus erythematosus and autoimmunity. J Autoimmun 2016;74:118-38.

7 Bertsias GK, Salmon JE, Boumpas DT. Therapeutic opportunities in systemic lupus erythematosus: state of the art and prospects for the new decade. Ann Rheum Dis 2010;69:1603-11.

8 Draborg A, Izarzugaza JMG, Houen G. How compelling are the data for Epstein-Barr virus being a trigger for systemic lupus and other autoimmune diseases? Curr Opin Rheumatol 2016;28:398-404.

9 Alfadhli SM. Genetics and epigenetic in systemic lupus erythematosus. In: Almoallim H (ed). Systemic lupus erythematosus. InTech, 2012.

10 Pathak S, Mohan C. Cellular and molecular pathogenesis of systemic lupus erythematosus: lessons from animal models. Arthritis Res Ther 2011;13:241.

11 Sabahi R, Anolik JH. B-cell-targeted therapy for systemic lupus erythematosus. Drugs 2006;66:1933-48.

12 Thomson K, Murphy A, Goodfield M, Misbah S. Is it useful to test for antibodies to extractable nuclear antigens in the presence of a negative antinuclear antibody on Hep-2 cells? J Clin Pathol 2001;54:413.

13 Abeles AM, Abeles M. The clinical utility of a positive antinuclear antibody test result. Am J Med 2013;126:342-8.

14 Egner W. The use of laboratory tests in the diagnosis of SLE. J Clin Pathol 2000;53:424-32.

15 Larosa M, Iaccarino L, Gatto M, Punzi L, Doria A. Advances in the diagnosis and classification of systemic lupus erythematosus. Expert Rev Clin Immunol 2016;8:1-12.

16 Buyon J, Furie R, Putterman C et al. Reduction in erythrocytebound complement activation products and titres of anti-Clq antibodies associate with clinical improvement in systemic lupus erythematosus. Lupus Sci Med 2016;3:e000165.

17 Rees F, Doherty M, Grainge MJ et al. Mortality in systemic lupus erythematosus in the United Kingdom 1999-2012. Rheumatology 2016;55:854-60.

18 Rees F, Doherty M, Grainge M et al. Burden of comorbidity in systemic lupus erythematosus in the UK, 1999-2012. Arthritis Care Res 2016;68:819-27.

19 Yee C-S, Farewell VT, Isenberg DA et al. The use of systemic lupus erythematosus disease activity index-2000 to define active disease and minimal clinically meaningful change based on data from a large cohort of systemic lupus erythematosus patients. Rheumatology 2011;50:982-8.

20 Fernando MMA. How to monitor SLE in routine clinical practice. Ann Rheum Dis 2005;64:524-7.

21 Al Sawah S, Zhang X, Zhu B et al. Effect of corticosteroid use by dose on the risk of developing organ damage over time in systemic lupus erythematosus-the Hopkins Lupus Cohort. Lupus Sci Med 2015;2:e000066.

22 Ruiz-Irastorza G, Garcia M, Espinosa G et al. First month prednisone dose predicts prednisone burden during the following 11 months: an observational study from the RELES cohort. Lupus Sci Med 2016;3:e000153.

23 Houssiau FA, Vasconcelos C, D'Cruz D et al. Immunosuppressive therapy in lupus nephritis: the Euro-Lupus Nephritis Trial, a randomized trial of low-dose versus high-dose intravenous cyclophosphamide. Arthritis Rheum 2002;46:2121-31.

24 Houssiau FA, D’Cruz D, Sangle S et al. Azathioprine versus mycophenolate mofetil for long-term immunosuppression in lupus nephritis: results from the MAINTAIN Nephritis Trial. Ann Rheum Dis 2010;69:2083-9.

25 Ruiz-Irastorza G, Ramos-Casals M, Brito-Zeron P, Khamashta MA. Clinical efficacy and side effects of antimalarials in systemic lupus erythematosus: a systematic review. Ann Rheum Dis 2010;69:20-8.

26 Furie R, Petri M, Zamani $\mathrm{O}$ et al. A phase III, randomized, placebocontrolled study of belimumab, a monoclonal antibody that inhibits B lymphocyte stimulator, in patients with systemic lupus erythematosus. Arthritis Rheum 2011;63:3918-30. 
27 Navarra SV, Guzmán RM, Gallacher AE et al. Efficacy and safety of belimumab in patients with active systemic lupus erythematosus: a randomised, placebo-controlled, phase 3 trial. Lancet 2011;377:721-31.

28 Khamashta M, Merrill JT, Werth VP et al. Sifalimumab, an antiinterferon- monoclonal antibody, in moderate to severe systemic lupus erythematosus: a randomised, double-blind, placebocontrolled study. Ann Rheum Dis 2016;75:1909-16.

29 National Institute for Health and Care Excellence. Belimumab for treating active autoantibody-positive systemic lupus erythematosus. NICE technology appraisal No 397. London: NICE, 2016.

30 Rovin BH, Furie R, Latinis K et al. Efficacy and safety of rituximab in patients with active proliferative lupus nephritis: the lupus nephritis assessment with rituximab study. Arthritis Rheum 2012;64:1215-26.

31 Merrill JT, Neuwelt CM, Wallace DJ et al. Efficacy and safety of rituximab in moderately-to-severely active systemic lupus erythematosus: the randomized, double-blind, phase ii/iii systemic lupus erythematosus evaluation of rituximab trial. Arthritis Rheum 2010;62:222-33.
32 Leandro MJ, Cambridge G, Edwards JC, Ehrenstein MR, Isenberg DA. B-cell depletion in the treatment of patients with systemic lupus erythematosus: a longitudinal analysis of 24 patients. Rheumatology 2005;44:1542-5.

33 Leandro MJ, Edwards JC, Cambridge G, Ehrenstein MR, Isenberg DA. An open study of B lymphocyte depletion in systemic lupus erythematosus. Arthritis Rheum 2002;46:2673-7.

34 Smith KGC, Jones RB, Burns SM, Jayne DRW. Long-term comparison of rituximab treatment for refractory systemic lupus erythematosus and vasculitis: remission, relapse and re-treatment. Arthritis Rheum 2006;54:2970-82.

Address for correspondence: Dr M Shaikh, Department of Rheumatology, Addenbrooke's Hospital, Cambridge University Hospitals NHS Foundation Trust, Hills Road, Cambridge CB2 0QQ, UK.

Email: malihashaikh@doctors.org.uk 